\title{
Successful Treatment of Fulminant Myocarditis
}

\section{Calvin E Tucker ${ }^{1 *}$, Margaret J Fernandez ${ }^{2}$ and Christopher C Morrison ${ }^{3,4}$}

${ }^{1}$ Clinical Pharmacist, Critical Care St. Vincent's Medical Center Jacksonville FL, USA

${ }^{2}$ Cardiothoracic/Surgical Intensive Care Unit Clinical Pharmacist, Jackson Memorial Hospital, Miami, FL, USA

${ }^{3}$ Neuroscience Intensive Care Unit Clinical Pharmacist, Jackson Memorial Hospital, Miami, FL, USA

${ }^{4}$ Voluntary Clinical Assistant Professor, University of Miami Miller School of Medicine, Miami, FL, USA

\begin{abstract}
Purpose: Myocarditis is an inflammatory process of the myocardium that can have an assortment of different etiologies such as infection, systemic disease and/or exposure to medications and toxins. There can be a variety of clinical presentations ranging from mild dyspnea and chest pain that can be self-limiting to cardiogenic shock and death. Although myocarditis can have several causes, viral infection remains the leading cause of both acute and chronic myocarditis. Corticosteroids, Intravenous Immunoglobulin (IVIG) and ventricular assist devices are controversial treatment options with minimal evidence of efficacy in the adult population.
\end{abstract}

Summary: A critically ill patient presented to a large academic healthcare facility with flu-like symptoms and severe left ventricular dysfunction. The patient was given broad spectrum antimicrobials, corticosteroids and intravenous immunoglobulin as part of their treatment regimen. Due to severe left ventricular dysfunction, the patient required the use of a left ventricular assist device and extracorporeal membrane oxygenation for cardiac and hemodynamic support. After improvement in left ventricular function, the patient experience neuropathies requiring physical therapy and rehabilitation.

Conclusion: This case report presents a patient with severe left ventricular dysfunction due to myocarditis. After administration of broad antimicrobial coverage, corticosteroids and IVIG, our patient experienced recovery of left ventricular function and overall improvement in his condition.

Keywords: Extracorporeal membrane; Intravenous immunoglobulin; Left ventricular dysfunction; Myocarditis; Treatment; Viral infection

\section{Introduction}

Myocarditis is an inflammatory process of the myocardium that can have an assortment of different etiologies such as infection, systemic disease and/or exposure to medications and toxins [1]. There are a variety of clinical presentations ranging from mild dyspnea and chest pain that can be self-limiting to cardiogenic shock and death [2]. Although there can be several causes, viral infection remains the leading cause of both acute and chronic myocarditis [2,3]. Common viral infections associated with infection of the myocardium are the adenoviruses and Coxackievirus B, although other viruses have been associated with myocarditis such as Human Immunodeficiency Virus (HIV), Cytomegalovirus (CMV), Epstein-Barr Virus (EBV) and Herpes Simplex Virus (HSV) [2,4]. The use of Intravenous Immunoglobulin (IVIG) in the treatment of acute myocarditis is controversial, with proven efficacy in the pediatric population and minimal literature showing benefit in adults [5]. IVIG failed to consistently show significant improvement in left ventricular function or functional capacity of adult patients in randomized controlled trials [6]. High dose steroids have exhibited limited success in certain types of myocarditis, however an optimum treatment regimen is not clearly defined [3]. Myocarditis can cause severe hemodynamic comprise that may not be responsive to medical therapy. Mechanical circulatory support provides a reasonable treatment for acute circulatory collapse [5]. In the present case we describe a critically ill patient diagnosed with fulminant myocarditis treated with IVIG, high dose corticosteroids, broad spectrum antibiotics and requirement of mechanical assist devices resulting in resolution of left ventricular dysfunction and clinical improvement.

\section{Case Report}

Our patient is a 31 year old, Hispanic male with no significant medical history, who presented to his primary care physician complaining of flu-like symptoms including sore throat, fever, nausea, vomiting, diarrhea, fatigue and myalgias. Over the next four days he was given two intramuscular injections of 1 gram ceftriaxone and started on azithromycin $250 \mathrm{mg}$ PO daily for 5 days with no resolution of symptoms. He then presented to a local community hospital for persistent flu-like symptoms. Upon examination he was found to have bibasilar pneumonia, acute renal failure and an elevated White Blood Cell Count (WBC). Empiric broad spectrum antibiotics were started with vancomycin 1 gram IV every 24 hours and piperacillintazobactam 3.375 gram IV every 8 hours. The patient's condition continued to deteriorate to the extent he became hypotensive requiring $20 \mathrm{mcg} / \mathrm{kg} / \mathrm{min}$ of dopamine and $5 \mathrm{mcg} / \mathrm{kg} / \mathrm{min}$ of dobutamine. An echocardiogram was performed showing moderately reduced left ventricular function with an ejection fraction of $35 \%$ and it was at this time the patient was transferred to the Cardiac Care Unit (CCU) at Jackson Memorial Hospital (JMH).

Upon admission to the CCU, the serum creatinine was acutely elevated to $5.24 \mathrm{mg} / \mathrm{dL}$, suggesting acute renal failure and required a consult to the Nephrology service. Upon auscultation the patient was found to have bilateral rales and ronchi but not wheezing. A chest radiograph was obtained that initially showed right lower lobe opacity suggestive of pneumonia, interstitial pulmonary edema and a right pleural effusion. Due to worsening respiratory status the patient was sedated and intubated. His respiratory status continued to worsen despite being on aggressive ventilator settings. In order to increase lung compliance and improve ventilation the patient was paralyzed with

${ }^{*}$ Corresponding author: Calvin E Tucker, Clinical Pharmacist, Critical Care, St. Vincent's Medical Center, Jacksonville, FL, USA, E-mail: Calvin.Tucker@jaxhealth.com

Received January 04, 2013; Accepted February 21, 2013; Published February 23, 2013

Citation: Tucker CE, Fernandez MJ, Morrison CC (2013) Successful Treatment of Fulminant Myocarditis. J Clin Case Rep 3: 256. doi:10.4172/2165-7920.1000256

Copyright: (c) 2013 Tucker CE, et al. This is an open-access article distributed under the terms of the Creative Commons Attribution License, which permits unrestricted use, distribution, and reproduction in any medium, provided the original author and source are credited. 
vecuronium at $1 \mathrm{mcg} / \mathrm{kg} / \mathrm{min}$. To increase the spectrum of coverage due to the patients rapid deterioration, the antibiotics were changed to meropenem $500 \mathrm{mg}$ IV every 6 hours, vancomycin $1.5 \mathrm{~g}$ IV every 24 hours and azithromycin $500 \mathrm{mg}$ IV every 24 hours. Dobutamine 5 $\mathrm{mcg} / \mathrm{kg} / \mathrm{min}$ and dopamine $20 \mathrm{mcg} / \mathrm{kg} / \mathrm{min}$ were continued although the patient continued to be hypotensive. Vasopressin $1.2 \mathrm{units} / \mathrm{hr}$ and phenylephrine $350 \mathrm{mcg} / \mathrm{min}$ was added to increase Mean Arterial Pressure (MAP). A repeat echocardiogram was performed showing severely reduced left ventricular function and an ejection fraction of 20 percent. Infectious Diseases Service was consulted and began a complete work up for acute myocarditis. An endomyocardial biopsy was performed on day one and day four, along with blood, urine and Bronchaveolar Lavage (BAL) cultures. Virology tests included; Coxsackievirus, CMV, HIV, Influenza, Hepatitis, Dengue, EBV, Parvovirus, and Borrelia Burgdorferi (Lyme disease). Due to the suspicion of viral infection oseltamivir $75 \mathrm{mg}$ PO twice daily and acyclovir $700 \mathrm{mg}$ IV every 48 hours was started. Methylprednisolone $125 \mathrm{mg}$ IV every 6 hours and Intravenous Immunoglobulin (IVIG) $1 \mathrm{~g} / \mathrm{kg}$ for three doses were started for suspected myocarditis. Biopsy results from day one revealed inflammatory and mononuclear infiltrates consistent with viral myocarditis. The biopsy from day four revealed inflammatory changes that were more advanced than the first biopsy. As culture results were finalized, vancomycin, meropenem and azithromycin were discontinued after five days of treatment. Oseltamivir and acyclovir were continued for a total of 14 days.

Despite the use of pharmacologic therapy such as inotropes, vasopressors and broad spectrum antimicrobial coverage the patient continued to be hypotensive and showed evidence of hypoperfusion. A Left Ventricular Assist Device (LVAD), Impella ${ }^{\circledR} 2.5$, was inserted to provide additional hemodynamic support on day four of admission to JMH. Less than 24 hours after the insertion of the Impella ${ }^{\circledR} 2.5$, the patient's condition continued to worsen exhibited by persistent hypotension and metabolic acidosis with a $\mathrm{pH}$ of 6.93 and lactic acid of $11.3 \mathrm{mmol} / \mathrm{L}$. The patient was transferred to the surgical intensive care unit and cardiothoracic surgery was consulted for insertion of Extracorporeal Membrane Oxygenation (ECMO) to provide complete cardiac and pulmonary support. The use of ECMO was able to provide adequate hemodynamic support, allowing the Impella ${ }^{\circledR} 2.5$ to be removed 48 hours later. Shortly after the initiation of ECMO, the patient began to experience oral and nasal bleeding. He was treated with vitamin K $10 \mathrm{mg}$ subcutaneous every 8 hours for 3 doses and multiple units of fresh frozen plasma and platelets over the next 48 hours. Otolaryngology (ENT) and Gastroenterology were consulted to evaluate the bleeding. Both services were unable to determine a source of bleeding and concluded the patient may have an underlying coagulopathy. No therapy was recommended as bleeding had resolved. Three days after initiation of ECMO, the patient's cardiac function began to improve, allowing for its discontinuation. Shortly after mechanical ventilation was decreased and the patient was extubated. Vasopressor and inotrope support were also weaned off.

In spite of a very complicated hospital course that included acute viral myocarditis, pronounced left ventricular dysfunction, pharyngeal bleed, multi-organ failure and severe acidosis requiring ECMO, eight days after being admitted to JMH the patient's left ventricular function recovered with an ejection fraction of 50 percent. Ten days after extubation the patient reported the inability to move his extremities, except his lower right leg. Neurology was consulted to evaluate the patient for the generalized weakness, which was determined to be critical illness polyneuropathy and myopathy. The patient progressively regained muscle strength and movement in his extremities with resolution of renal failure. Five weeks after being admitted to $\mathrm{JMH}$, the patient was transferred from the intensive care unit to the adult neurology rehabilitation floor.

\section{Discussion}

The pathophysiology of myocarditis involves both direct and virally mediated myocyte dysfunction and immune-related tissue injury. The immune system activation protects against virally mediated myocyte injury, however the immune response also contributes to the development of myocardial inflammation, necrosis and ventricular dysfunction [1]. The gold standard for the diagnosis of acute myocarditis and how to differentiate infectious from non-infectious causes remains unclear. According to the standard Dallas pathological criteria, myocarditis requires an inflammatory cellular infiltrate be present on endomyocardial biopsy; however studies have shown that the biopsy results on approximately one-half of patients with acute myocarditis do not have isolation of an organism from cardiac tissue. Molecular viral detection can potentially improve sensitivity of endomyocardial biopsy but the specificity of biopsy results remains unclear [2,3]. Our patient had endomyocardial biopsies performed; which had changes indicative of myocarditis. The patient was admitted to the hospital soon after experiencing flu-like symptoms. The time from symptom onset to hospital admission was about 5 days and endomyocardial biopsies are the most successful when performed as close as possible to symptom onset [2].

Patients with acute myocarditis can present with hemodynamic instability and left ventricular dysfunction that can progress to cardiogenic shock similar to patients with heart failure. First line treatment is mainly supportive care. Patients often show improvement with a standard heart failure regimen such as angiotensin-converting enzyme inhibitors, beta-blockers and diuretics [1,2]. If the left ventricular dysfunction is severe, vasopressors and inotropes may be required to help maintain cardiac output. If the patient's condition continues to deteriorate despite maximizing medical therapy or with severe left ventricular dysfunction, mechanical support such as LVAD or ECMO may be necessary to support cardiac output as a bridge to recovery or heart transplantation [2]. The uses of LVADs were designed to provide hemodynamic support in patients with severe left ventricular dysfunction. The LVAD unloads blood from the left ventricle and propels it forward into the systemic vasculature. As a result, the oxygen demand on the myocardial tissue decreases which aids myocardial recovery. The Impella ${ }^{\circledR} 2.5$ is a small LVAD which is inserted into the left ventricle that unloads the ventricle and expels blood into the ascending aorta. The Impella ${ }^{\circledR} 2.5$ was designed for ease of use; as a result it can only provide partial support with a blood flow of $2.5 \mathrm{~L} / \mathrm{min}$ which approximately half of the body's normal capacity. It is approved by the Food and Drug Administration (FDA) for a total duration of 6 hours but has been used in some patients for as long as 2 weeks. The use of percutaneous LVAD devices are being used progressively more in high risk Percutaneous Coronary Intervention (PCI) procedures because they provide greater increases in cardiac output than IntraAortic Balloon Pumps (IABP) [7]. The IABP is limited in its ability to provide circulatory support and if left ventricular function is severely compromised, an IABP will not be effective [7]. The Impella ${ }^{\circledR} 2.5$ offers several advantages when compared to ECMO primarily being its ease of insertion in the catheterization laboratory. It doesn't require cardiopulmonary bypass and survival rates similar to those associated with other circulatory support devices [8]. The development and increase use of percutaneous LVAD devices has widened the spectrum of indications for their use; however, unlike ECMO, they do not offer 
respiratory support or right ventricular support [9]. Although these invasive procedures are associated with significant risks to the patient, they may be necessary to maintain cardiac output and perfusion $[10,11]$.

Experimental models suggest that IVIG may have therapeutic use in myocarditis. Treatment with high dose IVIG $(2 \mathrm{~g} / \mathrm{kg})$ is proposed to have immunomodulatory effects that are multifactorial such as neutralization of pathogens, reduction in inflammatory cytokines and antiviral effects. When used in pediatric acute myocarditis, IVIG resulted in improved left ventricular function and better survival. There are some trials in adults that have reported improvement of left ventricular function with the use of high dose IVIG but the literature is inconsistent $[1,2,6]$. The most effective dose when using IVIG for myocarditis is controversial. Most of the studies that have shown benefit with IVIG used the higher total dosing of $2 \mathrm{~g} / \mathrm{kg}$. Dosing of IVIG that was utilized in our patient was $1 \mathrm{~g} / \mathrm{kg}$ for three doses. Our patient also received corticosteroids, broad spectrum antimicrobial coverage; therefore we cannot say that one specific intervention was solely responsible for the improvement of the patient. There have been several studies that have evaluated the use of corticosteroid treatment in myocarditis, with minimal literature demonstrating a benefit $[3,4]$. Both corticosteroids and IVIG have anti-inflammatory properties that may be beneficial in acute myocarditis. The antiviral properties of IVIG make its use in our patient reasonable since it is theorized that our patient were suffering from acute viral myocarditis and despite negative viral cultures [12].

\section{Conclusion}

The present report describes a critically ill patient with acute myocarditis treated at JMH. Our patient had positive myocardial enzymes, severely reduced ejection fraction and evidence of inflammation on endomyocardial biopsy. After administration of broad spectrum antimicrobial coverage, corticosteroids, IVIG and the use of ventricular assist devices, our patient experienced recovery of their left ventricular function and overall improvement in condition.

\section{References}

1. Bowles NE, Vallejo J (2003) Viral causes of cardiac inflammation. Curr Opin Cardiol 18: 182-188.

2. Goland S, Czer LS, Siegel RJ, Tabak S, Jordan S, et al. (2008) Intravenous immunoglobulin treatment for acute fulminant inflammatory cardiomyopathy: series of six patients and review of literature. Can J Cardiol 24: 571-574.

3. Gupta S, Markham DW, Drazner MH, Mammen PP (2008) Fulminant myocarditis. Nat Clin Pract Cardiovasc Med 5: 693-706.

4. Cooper LT (2009) Myocarditis. N Engl J Med 360: 1526-1538.

5. Lin CT, Hsu PS, Chen BC, Hong GJ, Tsai CS (2010) Successful treatment of acute fulminant myocarditis with double sets of extracorporeal membrane oxygenation: report of a case and review of the literature. Thorac Cardiovasc Surg 58: 433-435.

6. McNamara DM, Holubkov R, Starling RC, Dec GW, Loh E, et al. (2001) Controlled trial of intravenous immune globulin in recent-onset dilated cardiomyopathy. Circulation 103: 2254-2259.

7. McCulloch B (2011) Use of the Impella 2.5 in high-risk percutaneous coronary intervention. Crit Care Nurse 31: e1-e16.

8. Chaparro SV, Badheka A, Marzouka GR, Tanawuttiwat T, Ahmed F, et al. (2012) Combined use of Impella left ventricular assist device and extracorporeal membrane oxygenation as a bridge to recovery in fulminant myocarditis. ASAIO J 58: 285-287.

9. Suradi H, Breall JA (2011) Successful use of the Impella device in giant cell myocarditis as a bridge to permanent left ventricular mechanical support. Tex Heart Inst J 38: 437-440.

10. Rose NR (2009) Myocarditis: Infection versus autoimmunity. J Clin Immunol 29: $730-737$.

11. Robinson J, Hartling L, Vandermeer B, Crumley E, Klassen TP (2005) Intravenous immunoglobulin for presumed viral myocarditis in children and adults. Cochrane Database Syst Rev 25: CD004370.

12. Schultz JC, Hilliard AA, Cooper LT Jr, Rihal CS (2009) Diagnosis and treatment of viral myocarditis. Mayo Clin Proc 84: 1001-1009. 